\title{
Allopurinol improves endothelial function in sleep apnoea: a randomised controlled
} study

\author{
A.A. El Solh*, R. Saliba*, T. Bosinski”, B.J.B. Grant*, E. Berbary* and N. Miller*
}

ABSTRACT: Increased oxidative stress in obstructive sleep apnoea is thought to contribute to endothelial dysfunction. The objective of this study was to test the hypothesis that inhibition of xanthine oxidase by allopurinol can improve endothelial function in patients with obstructive sleep apnoea.

A randomised double-blind placebo-controlled crossover study was performed on 12 patients with moderate-to-severe obstructive sleep apnoea, comparing $300 \mathrm{mg}$ allopurinol daily for 2 weeks with placebo. Endothelial function was assessed using hyperaemia-induced flowmediated vasodilation (FMD) at baseline and following treatment. Plasma malondialdehyde levels were compared in order to assess significant changes in oxidative stress.

Baseline FMD correlated significantly with the severity of sleep apnoea and the time spent with an arterial oxygen saturation of $<\mathbf{9 0} \%$. Allopurinol caused a significant increase in FMD compared to placebo $(10.4 \pm 3.2$ versus $7.4 \pm 2.8 \%$, respectively). Plasma malondialdehyde levels were significantly reduced with allopurinol treatment $\left(1.5 \pm 0.3\right.$ versus $\left.1.2 \pm 0.3 \mu \mathrm{mol} \cdot \mathrm{L}^{-1}\right)$, consistent with reduced oxidative stress.

Allopurinol improves endothelial dysfunction in patients with moderate-to-severe obstructive sleep apnoea. These observations suggest that xanthine oxidase contributes significantly to vasodilatory impairment.

KEYWORDS: Allopurinol, endothelium, free radicals, sleep apnoea growing body of evidence points to a causal association between obstructive sleep apnoea (OSA) and cardiovascular diseases, including hypertension, coronary artery disease and cerebrovascular accident [1-4]. Various mechanisms have been implicated in the pathogenesis of vasculopathy in patients with OSA. These mechanisms encompass numerous systems that involve neural, humoral, endocrine and inflammatory mediators. Regardless of the underlying illness, these syndromes reflect a persistent inciting injury leading to endothelial dysfunction and to a compromise of the integrity of the vascular endothelium as a consequence of an unregulated inflammatory response. Indeed, in awake patients with OSA and in the absence of any other disease state or potential cause of endothelial dysfunction, the endotheliummediated vasodilator response to intra-arterial acetylcholine is markedly impaired in comparison to closely matched subjects without OSA $[5,6]$.

Since OSA is characterised by repeated cycles of hypoxia reoxygenation, it was postulated that the hypoxia-activated enzyme xanthine oxidase would create a flux of reactive oxygen species, namely superoxide anions, capable of lipid peroxidation and protein degradation $[7,8]$. Perhaps more important is the potential of the superoxide anions to scavenge circulating nitric oxide, a potent vascular relaxing factor and ubiquitous signalling molecule in the human body. In order to reduce these harmful effects of oxidative stress, most work has concentrated on using antioxidant vitamins to negate the effect of superoxide anions. However, it may also be possible to use an alternative strategy of preventing the formation of superoxide anions. In the vascular endothelium, the xanthine oxidase system is one of the main producers of superoxide anions [9]. Xanthine oxidase inhibition with allopurinol would probably prevent the formation of superoxide free radicals, which could lead to better endothelial function [10]. In patients with heart failure and diabetes mellitus, an improvement in endothelial dysfunction was demonstrated after 1 month of treatment with allopurinol [11, 12]. Hence, the present

\section{AFFILIATIONS}

*Division of Pulmonary, Critical Care and Sleep Medicine, Dept of Medicine, School of Medicine and Biomedical Sciences and the Veterans Affairs Medical Center, and ${ }^{*}$ School of Pharmacy, University at Buffalo, Buffalo, NY, USA.

CORRESPONDENCE

A.A. El Solh

Division of Pulmonary Critical Care and Sleep Medicine Erie County Medical Center 462 Grider Street Buffalo NY 14215 USA Fax: 17168986139 E-mail: solh@buffalo.edu

Received:

August 272005 Accepted after revision: December 192005 
randomised double-blind placebo-controlled crossover study was conducted in order to investigate whether allopurinol, as a xanthine oxidase inhibitor, might improve endothelial dysfunction in patients recently diagnosed with moderate-tosevere OSA. Plasma malondialdehyde (MDA) levels were also compared in order to assess any change in oxidative stress following allopurinol and placebo treatment.

\section{METHODS}

\section{Study subjects}

The present study was approved by the Institutional Review Board of the University at Buffalo (Buffalo, NY, USA). Subjects aged $\geqslant 18$ yrs were considered for enrolment if they were able and willing to sign informed consent, showed documented moderate-to-severe OSA on the basis of overnight polysomnography [13], defined as an apnoea/hypopnoea index (AHI) of $\geqslant 15$ events $\cdot h^{-1}$, and were not receiving therapy for sleep apnoea. Subjects who met the following criteria were excluded from participation: 1) current smokers (or those who had quitted within 6 months prior to enrolment); 2) congestive heart failure (with an ejection fraction of $<40 \%$ by multiple uptake gated acquisition (blood pool scan) or two-dimensional echocardiography); 3) history of hypertension, diabetes mellitus, or gout; 4) allergic reaction to allopurinol or those receiving allopurinol; 5) presence of active malignancy; 6) use of vasodilators (including calcium channel blockers, nitrates, or angiotensin-converting enzyme inhibitors) and/or statins; 7) treatment with continuous positive airway pressure (CPAP) prior to or at the time of enrolment; 8) recent surgery (within 3 months prior to enrolment); 9) presence of active acute or chronic infection; and 10) use of steroidal and nonsteroidal anti-inflammatory agents for $>1$ week, irrespective of dose prescribed.

Following informed consent, all participants underwent clinical and metabolic evaluation, echocardiography and a vascular reactivity study. Subsequently, patients were randomly assigned (1:1) into the allopurinol (300 mg oral tablets daily) or placebo group for a period of 2 weeks intercalated by a 2-week wash-out period. Placebo comprised Avicel (FMC BioPolymer, Philadelphia, PA, USA), a microcrystalline cellulose commonly used in pharmaceutical manufacturing. The randomisation procedure was performed by a central pharmacy using sequentially numbered sealed opaque envelopes. A weekly telephone call was made to all participants in order to enquire about potential side-effects. At the end-of-treatment visit, the medication bottle was retrieved and the number of remaining pills counted. Both participants and investigators were blinded to the treatment provided.

\section{Vascular reactivity study}

Prior to the scheduled vascular reactivity study, participants were asked to refrain from caffeine intake and heavy exercise for $8 \mathrm{~h}$. Studies were conducted between 08:30 and 09:30 h in order to avoid the recognised diurnal fluctuations in endothelial function. The examination began with a 15-min rest period, with the subject lying supine in a quiet room. A standard paediatric blood pressure cuff was placed $5 \mathrm{~cm}$ below the right antecubital fossa. Blood pressure and cardiac frequency were recorded every $5 \mathrm{~min}$ using an automated sphygmomanometer on the left arm. The right brachial artery was identified $\sim 7 \mathrm{~cm}$ proximal to the brachial bifurcation using a $5.0-\mathrm{MHz}$ linear-array transducer (Acuson 128XP; Acuson, Mountain View, CA, USA). The transducer was positioned to allow simultaneous viewing of the blood adventitial interfaces on the near and far walls. A solid gel pad was used to optimise focal depth and minimise reverberations. When a satisfactory transducer position was found, the skin was marked and the arm remained in the same position throughout the duration of the study.

Resting brachial artery diameter was measured online using electronic callipers at end-diastole (peak of $\mathrm{R}$ wave of the echocardiogram). The mean of three readings was used. Thereafter, arterial occlusion was created by inflation of the pneumatic blood pressure cuff to a pressure of $50 \mathrm{mmHg}$ above baseline systolic blood pressure for $5 \mathrm{~min}$. This was followed by rapid deflation of the cuff to induce a brief highflow state through the brachial artery. Repeat measurement of brachial artery diameter was made 1 min after cuff deflation. Data were reported as baseline diameter, absolute change in diameter (maximum minus baseline) and percentage change in diameter (absolute change/baseline $\times 100$ ), known also as percentage flow-mediated vasodilation (FMD). All ultrasonographic studies were performed by the same operator, who was blinded to the severity of sleep-disordered breathing or treatment of the study subjects. The variability in baseline diameter measurements, expressed as intra-session variability, was determined at $0.6 \%$. A repeat vascular reactivity test was performed at baseline and at the end of each treatment period.

\section{Plasma malondialdehyde levels}

A venous blood sample was obtained before each vascular reactivity test. Plasma samples were kept in a liquid nitrogen tank until analysis. Plasma MDA levels were determined according to a previously reported method [14]. Briefly, 20- $\mu \mathrm{L}$ plasma samples were mixed with $4.0 \mathrm{~mL} \mathrm{~N} / 12$ sulphuric acid and then $0.5 \mathrm{~mL} 10 \%$ phosphotungstic acid were added. After centrifuging the mixture for $10 \mathrm{~min}$ at $1,400 \times g$, the supernatant was discarded and the sediment mixed with $2.0 \mathrm{~mL} \mathrm{~N} /$ 12 sulphuric acid and $0.3 \mathrm{~mL} 10 \%$ phosphotungstic acid. The mixture was again centrifuged for $10 \mathrm{~min}$ at $1,400 \times g$ and the sediment suspended in $4.0 \mathrm{~mL}$ distilled water and then $1.0 \mathrm{~mL}$ thiobarbituric acid reagent (a mixture of equal volumes of $0.67 \%$ thiobarbituric acid aqueous solution and glacial acetic acid) was added. The reaction mixture was heated for $60 \mathrm{~min}$ at $95^{\circ} \mathrm{C}$. After cooling with tap water, $5.0 \mathrm{~mL} n$-butanol were added. After centrifugation for $15 \mathrm{~min}$ at $1,400 \times g$, the $n$ butanol layer was removed for fluorometric measurement at $553 \mathrm{~nm}$ with 515-nm excitation.

\section{Statistical analysis}

A pre-study power calculation using an $\alpha$ of 0.05 , a $\beta$ of 0.10 and an estimated clinical significance of a $25 \%$ change in FMD between baseline and treatment found that a sample size of 12 was necessary in order to find a difference.

Data are presented as mean $\pm \mathrm{SD}$. Comparison between treatments was performed by means of repeated-measures ANOVA with Bonferroni correction for multiple comparisons for within-group effects and order of treatment periods. A paired t-test was used when appropriate. Correlations between FMD and continuous variables were obtained using 
Spearman's rank test. Cook's distance was used to assess potential outliers. All reported p-values were two-tailed. The level of significance was set at $5 \%$.

\section{RESULTS}

\section{Patient characteristics}

Between June 2004 and May 2005, all 12 patients (seven males and five females) who agreed to participate signed their informed consent prior to enrolment. None of the patients withdrew during the study period and there were no adverse events reported due to allopurinol or placebo treatment. The baseline characteristics of the study population are shown in table 1. Eight patients were obese (body mass index (BMI) $\geqslant 30 \mathrm{~kg} \cdot \mathrm{m}^{-2}$ ), three were overweight $\left(\mathrm{BMI} \geqslant 25 \mathrm{~kg} \cdot \mathrm{m}^{-2}\right.$ but $<30 \mathrm{~kg} \cdot \mathrm{m}^{-2}$ ) and one patient was in the normal range $\left(\right.$ BMI $\left.<25 \mathrm{~kg} \cdot \mathrm{m}^{-2}\right)$. All patients exhibited normal renal function. Four showed evidence of hypercholesterolaemia (>200 mg. $\mathrm{dL}^{-1}$ ) but none were receiving any medical treatment. The polysomnographic characteristics are presented in table 2 . The AHI was $>30$ events $\cdot h^{-1}$ in $83 \%$ and $\geqslant 60$ events $\cdot h^{-1}$ in $50 \%$ of the population.

\section{Vascular reactivity study}

There was no difference in endothelium-dependent vasodilation between baseline and placebo treatment $(6.9 \pm 2.4$ versus $7.4 \pm 2.8 \%$, respectively; $p=0.9 ;$ fig. $1 \mathrm{a})$, but there was a significant improvement in FMD after allopurinol treatment compared to baseline $(6.9 \pm 2.4$ versus $10.4 \pm 3.2 \%$; $<<0.001$;

\section{TABLE 1 Characteristics of the study population}

$\begin{array}{lc}\text { Demographics } & \\ \text { Subjects } \mathrm{n} & 12 \\ \text { Age yrs } & 47.3 \pm 8.7(29-60) \\ \text { Body mass index } \mathrm{kg} \cdot \mathrm{m}^{-2} & 39.1 \pm 13.6(23-67) \\ \text { Systolic blood pressure } \mathrm{mmHg} & 126 \pm 5(115-136) \\ \text { Diastolic blood pressure } \mathrm{mmHg} & 76 \pm 3(68-86) \\ \text { Metabolic profile } & \\ \text { Total cholesterol } \mathrm{mg} \cdot \mathrm{dL}^{-1} & 201 \pm 35(164-276) \\ \mathrm{LDL} \text { cholesterol } \mathrm{mg} \cdot \mathrm{dL}^{-1} & 118 \pm 27(69-174) \\ \mathrm{HDL} \text { cholesterol } \mathrm{mg} \cdot \mathrm{dL}^{-1} & 49 \pm 18(32-93) \\ \text { Glucose } \mathrm{mg} \cdot \mathrm{dL}^{-1} & 98 \pm 15(77-121) \\ \text { Uric acid } \mathrm{mg} \cdot \mathrm{dL}^{-1} & 4.6 \pm 0.9(3.2-6.4) \\ \text { Left ventricular ejection fraction \% } & 59.6 \pm 4.9(50-70)\end{array}$

Data are presented as mean \pm SD (range), unless otherwise stated. LDL: lowdensity lipoprotein; HDL: high-density lipoprotein.

TABLE 2 Polysomnographic data of the study population

\section{Subjects $n$}

Apnoea/hypopnoea index events $\cdot h^{-1}$

Lowest arterial oxygen saturation \%

Time spent at arterial oxygen saturation of $<\mathbf{9 0 \%} \mathrm{min}$

Oxygen desaturation index events $\cdot h^{-1}$

Arousal index events $\cdot h^{-1}$

Data are presented as mean \pm SD (range), unless otherwise stated.
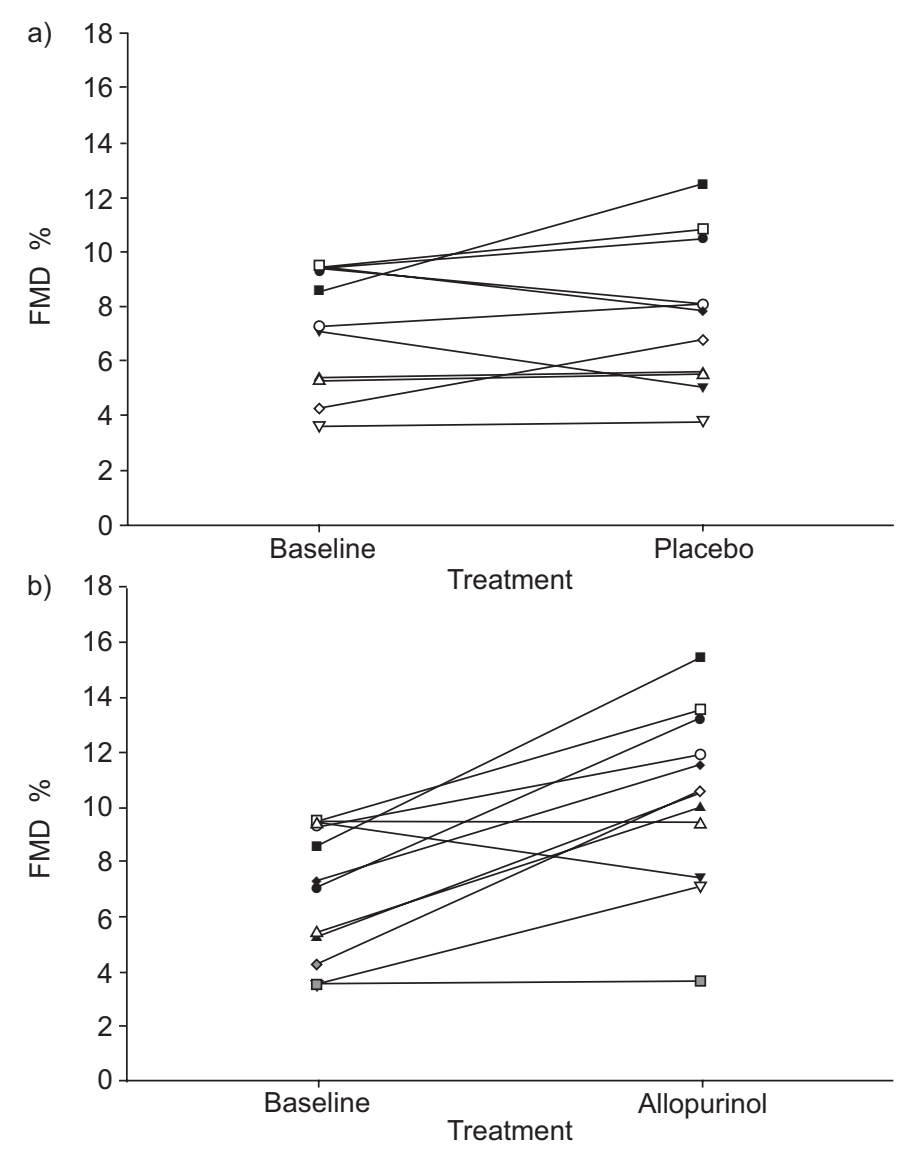

FIGURE 1. Flow-mediated vasodilation (FMD) of brachial artery in 12 subjects with obstructive sleep apnoea at baseline and after 2 weeks of treatment with a) placebo $(p=0.9)$ and b) allopurinol $(p<0.001)$. Different symbols represent individual patients.

fig. 1b). Similarly, the difference in FMD between placebo and allopurinol at the end of treatment remained significant $(7.4 \pm 2.8$ versus $10.4 \pm 3.2 \% ; p=0.001)$. Three subjects failed to show an improvement in FMD following allopurinol therapy. There were no common demographic characteristics or outstanding clinical values that would distinguish responders from nonresponders. The BMI of these three participants ranged $23-41 \mathrm{~kg} \cdot \mathrm{m}^{-2}$. One exhibited moderate (AHI 18) and two severe (AHI 38 and 75) OSA. MDA levels also failed to show a corresponding improvement between baseline and allopurinol therapy.

Substitution of placebo with allopurinol in six patients resulted in a significant improvement in FMD (placebo $7.9 \pm 3.2 \%$ versus allopurinol $10.5 \pm 3.8 \%$; $p=0.01$ ), whereas administration of placebo following allopurinol in the other six participants resulted in the FMD returning to baseline levels $(10.2 \pm 2.8 \%$ on allopurinol versus $6.8 \pm 2.4 \%$ on placebo; $p=0.07$ ).

Correlation analysis showed significant associations between baseline FMD and AHI $(r=-0.65, p=0.02)$, oxygen desaturation index $(\mathrm{ODI})(\mathrm{r}=-0.68, \mathrm{p}=0.01)$ and time spent with an arterial oxygen saturation of $<90 \%(r=-0.59, p=0.04)$, but not with nadir oxygen saturation $(\mathrm{r}=0.29, \mathrm{p}=0.2)$ or arousal index $(\mathrm{r}=-0.25, \mathrm{p}=0.3)$. There was also no correlation between FMD and age, BMI or total cholesterol level. 


\section{Plasma uric acid and malondialdehyde levels}

Allopurinol exerted the expected effect on plasma urate concentration (baseline $4.6 \pm 0.9 \mathrm{mg} \cdot \mathrm{dL}^{-1}$; placebo $4.5 \pm 0.7 \mathrm{mg}$. $\mathrm{dL}^{-1}$; allopurinol $\left.2.8 \pm 0.5 \mathrm{mg} \cdot \mathrm{dL}^{-1} ; \mathrm{p}<0.001\right)$. Similarly, there was a significant difference between MDA levels at baseline and following allopurinol therapy $(1.6 \pm 0.4$ versus $\left.1.2 \pm 0.3 \mu \mathrm{mol} \cdot \mathrm{L}^{-1} ; \mathrm{p}=0.002\right)$. The difference remained significant when placebo and allopurinol MDA levels were compared $\left(1.5 \pm 0.3\right.$ versus $1.2 \pm 0.3 \mu \mathrm{mol} \cdot \mathrm{L}^{-1}$, respectively; $\left.\mathrm{p}=0.008\right)$, but not when baseline and placebo levels were analysed $(1.6 \pm 0.4$ versus $1.5 \pm 0.3 \mu \mathrm{mol} \cdot \mathrm{L}^{-1}$, respectively; $\mathrm{p}=0.8$; fig. 2 ). Interestingly, MDA levels at baseline showed an inverse correlation with baseline FMD measurements, although the trend fell short of significance $(\mathrm{r}=0.4 ; \mathrm{p}=0.17)$. In contrast, there was a strong correlation between ODI and baseline MDA levels $(\mathrm{r}=0.7 ; \mathrm{p}=0.01$; fig. 3$)$. Furthermore, the changes in uric acid concentration and FMD between baseline and following allopurinol treatment correlated significantly $(r=0.6 ; p=0.05)$.

\section{DISCUSSION}

The results of the present study show that: 1) endothelial dysfunction correlates with severity of OSA; 2) impairment of
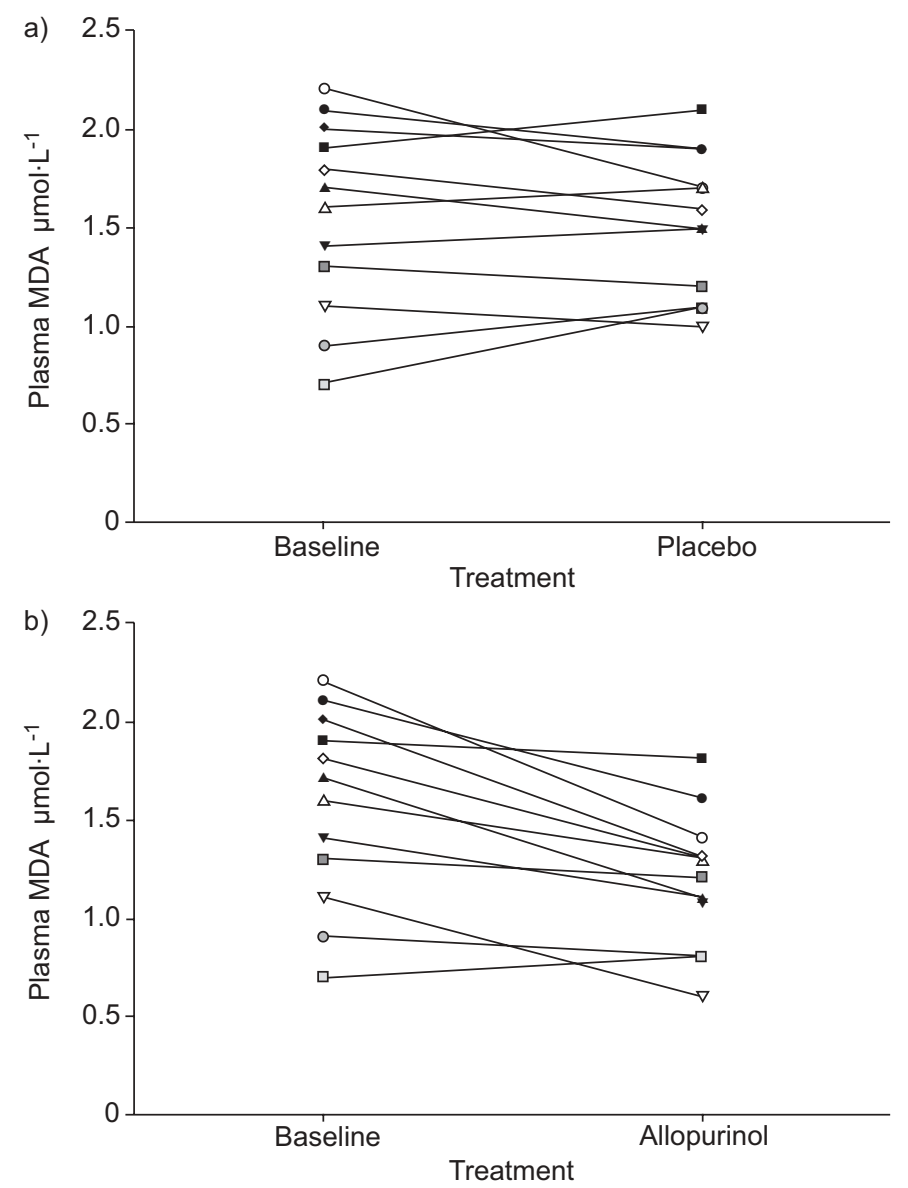

FIGURE 2. Differences in malondialdehyde (MDA) concentration between baseline and treatment with a) placebo $(p=0.8)$ and b) allopurinol $(p=0.002)$.

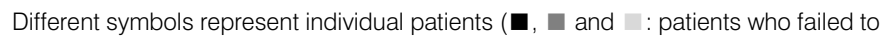
show an improvement in flow-mediated vasodilation after allopurinol therapy $(n=3))$.

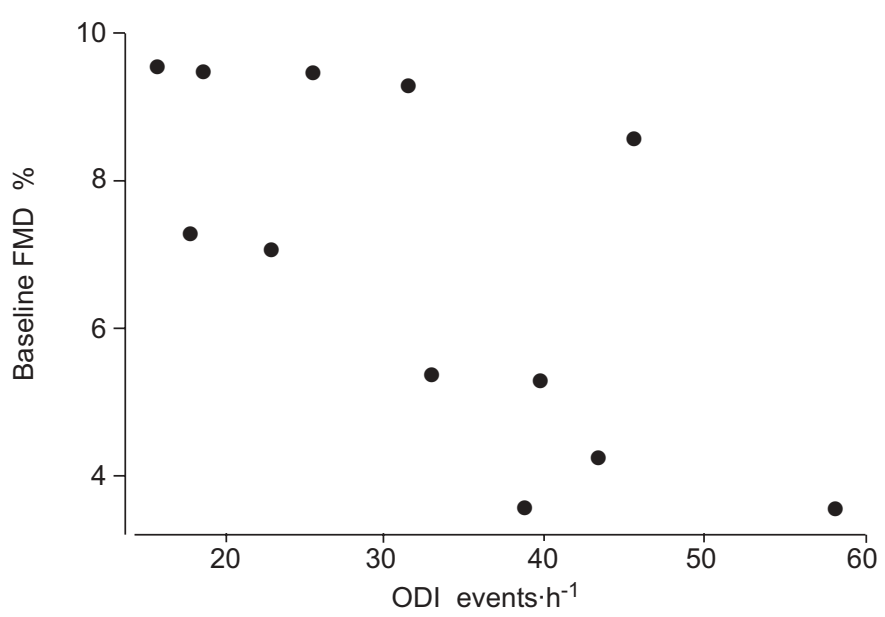

FIGURE 3. Scatter plot showing correlation between oxygen desaturation index (ODI) and baseline flow-mediated vasodilation (FMD) $(r=0.7 ; p=0.01)$

FMD is reversed with allopurinol; and 3) improvement in FMD following allopurinol therapy is associated with reduced oxidative stress.

Since the 1990s, there has been a rapid accumulation of evidence indicating links between sleep apnoea syndrome and cardiovascular morbidity and mortality. The fact that several studies have shown a dose-response relationship between the severity of breathing disorders during sleep and cardiovascular morbid conditions $[1,3,4,15,16]$ suggests that the link between cardiovascular disease and sleep apnoea is specific, and most probably related to some of the abnormalities which are unique to the syndrome. One of the underlying mechanisms that has received much attention in the recent literature is the state of the dysfunctional endothelium in patients with OSA. Endothelial dysfunction is considered to be a subclinical indicator of myocardial or vascular dysfunction before the emergence of clinical signs of overt cardiovascular disease [17]. Earlier studies reported that endothelial function in resistance vessels of the forearm was not only impaired in OSA patients in comparison with healthy subjects [18] but also showed a blunted vasodilation response to acetylcholine [5]. More recently, these findings were confirmed in a large communitybased sample of general healthy older individuals [19]. Using an ultrasound Doppler method similar to the present one, impairment of FMD in the brachial artery was also reported to correlate with AHI, an arterial oxygen saturation of $<90 \%$ and arousal index [20]. This association was independent of the presence of hypertension, diabetes and congestive heart failure.

The current study shows, for the first time, that inhibition of xanthine oxidase with allopurinol improves endothelial function in patients with OSA. Although the activity of xanthine oxidase has not been investigated in this group of patients, in vitro work has revealed that, in cultured human aortic endothelial cells, xanthine oxidase is the major source of free radicals and that hypoxanthine and xanthine oxidase increase endothelial dysfunction, which can then be improved using allopurinol [21]. Studies performed in subjects with diabetes mellitus and heart failure have found a parallel improvement 
in endothelial function with the administration of oral allopurinol for 1 month [11, 12]. Similar results were documented in patients with hypercholesterolaemia after receiving intravenous oxypurinol [22]. Since four of the participants showed evidence of elevated cholesterol levels, this could prompt the conclusion that the amelioration of FMD could be a manifestation of attenuated hypercholesterolaemia-associated superoxide anion generation [23]. However, review of these trials revealed that the cut-off for the definition of hypercholesterolaemia used in the present study $\left(>200 \mathrm{mg} \cdot \mathrm{dL}^{-1}\right)$ was lower than that used in comparable studies, in which a level of $>250 \mathrm{mg} \cdot \mathrm{dL}^{-1}$ was considered. Using the latter definition, only one patient in the present study would have met the criteria for hypercholesterolaemia. Furthermore, the strong correlation between ODI and baseline MDA levels emphasises the load of free radicals released due to OSA.

Given that the majority of the present study population were obese, it should be mentioned that obesity per se has been found to be independently associated with endothelial dysfunction in humans $[24,25]$. Clinical evidence from recent studies indicates that the increase in acetylcholine-mediated blood flow correlates inversely with BMI and waist-to-hip ratio [26]. This association is further complicated by the fact that insulin sensitivity is partly determined by the ability of the endothelium to produce nitric oxide. Comparing obese females to lean ones, DE JONGH et al. [27] found that there was a coupling between impaired capillary recruitment and decreased acetylcholine-mediated vasodilation during hyperinsulinaemia, and that these two entities correlated positively with insulin sensitivity. Although the interaction between obesity, insulin resistance and response to allopurinol were not assessed in the present study, the individual impact of these factors should be considered when interpreting the results.

Having recognised these confounding factors, a likely mechanism for the observed improvement in endothelium-dependent vasodilation in OSA subjects following xanthine oxidase inhibition is that allopurinol indirectly increases the in vivo bioavailability of endothelium-derived relaxing factors such as nitric oxide, presumably by blocking the production of reactive oxygen species mediated by xanthine oxidase. Studies of spontaneously hypertensive rats showed that recombinant forms of superoxide dismutase lowered blood pressure in these animals, in a similar manner to when the xanthine oxidase inhibitor oxypurinol [28] was administered. In humans with OSA, levels of plasma derivatives of nitric oxide were found to be significantly reduced and readily reversed with CPAP therapy $[29,30]$. The present findings of a significant reduction in plasma MDA concentration after allopurinol therapy further substantiate the above hypothesis. MDA levels were reduced only in those who received allopurinol treatment and not in those who received placebo. This reduction of oxidative stress is in agreement with the observed reduced susceptibility of low-density lipoprotein to oxidation following nasal CPAP therapy in patients with OSA [31].

The strength of the present study lies in its prospective randomised crossover design, which would minimise the presence of confounding variables and eliminate inherent individual variations in terms of generation of free radicals, hyperaemic vascular reactivity or response to treatment.
Moreover, this study has important clinical implications. Clinical trials with cardioprotective drugs, such as statins, angiotensin-converting enzyme inhibitors or spironolactone $[32,33]$, would suggest that the beneficial effects seen on endothelial function in the brachial artery of patients with OSA may be mirrored by similar changes in the cardiovascular system and may be associated with reduced cardiovascular events. Potential limitations worth mentioning include the relatively small sample size, as a result of the stringent inclusion and exclusion criteria used for participation. Secondly, a healthy control group were not included in the design. However, published reference values for FMD in healthy volunteers [34] were higher than those recorded in the present study population at baseline (FMD 8.6 \pm 4.6 versus $6.9 \pm 2.4 \%$, respectively), whereas reference values for MDA levels [35] were lower (0.765 versus $1.6 \pm 0.4 \mu \mathrm{mol} \cdot \mathrm{L}^{-1}$, respectively), confirming the presence of endothelial dysfunction and an increased burden of oxidative stress in OSA. Thirdly, there is the possibility of a carry-over effect of allopurinol from the treatment to the placebo phase. Although this effect could not be completely ignored in the absence of a repeat baseline FMD measurement prior to the second treatment, the relatively short half-life of allopurinol of $<3 \mathrm{~h}$ compared to the wash-out period of 2 weeks and the deterioration in vascular reactivity following withdrawal of allopurinol argue against this possibility. Fourthly, it has to be mentioned that the duration of therapy in the current investigation was chosen arbitrarily. However, improvement in endothelial function after allopurinol therapy has been reported after 1 day to 1 month of therapy $[12,36]$. Further studies are needed to determine the optimal dose and duration of allopurinol in reversing endothelial dysfunction and whether supplemental therapy with allopurinol in addition to CPAP therapy would provide further reduction in markers of cardiovascular diseases.

In conclusion, the present study shows that allopurinol improves endothelial function in patients with obstructive sleep apnoea. A probable mechanism is that allopurinol decreases free radical generation through inhibition of the xanthine oxidase system. The lower free radical burden improves the availability of nitric oxide, leading to improvement in endothelial vasodilation.

\section{ACKNOWLEDGEMENTS}

The authors thank the Erie County Medical Center (Buffalo, NY, USA) and all participants for their willingness to participate, and E. Ten Brock and L. Pineda (both Erie County Medical Center) for assisting in referral of patients to the Western New York Respiratory Research Center (Buffalo, NY, USA) for evaluation.

\section{REFERENCES}

1 Peker Y, Hedner J, Norum J, Kraiczi H, Carlson J. Increased incidence of cardiovascular disease in middle aged men with obstructive sleep apnea. Am J Respir Crit Care Med 2002; 166: 159-165.

2 Leung RS, Bradley TD. Sleep apnea and cardiovascular disease. Am J Respir Crit Care Med 2001; 164: 2147-2165.

3 Marin JM, Carrizo SJ, Vicente E, Agusti AG. Long-term cardiovascular outcomes in men with obstructive sleep 
apnoea-hypopnoea with or without treatment with continuous positive airway pressure: an observational study. Lancet 2005; 365: 1046-1053.

4 Gami AS, Howard DE, Olson EJ, Somers VK. Day-night pattern of sudden death in obstructive sleep apnea. $N$ Engl J Med 2005; 352: 1206-1214.

5 Kato M, Roberts-Thomson P, Phillips B, et al. Impairment of endothelium-dependent vasodilation of resistance vessels in patients with obstructive sleep apnea. Circulation 2000; 102: 2607-2610.

6 Kraiczi H, Hedner J, Peker Y, Carlson J. Increased vasoconstrictor sensitivity in obstructive sleep apnea. J Appl Physiol 2000; 89: 493-498.

7 Jessup W, Bedwell S, Dean R. The action of defined oxygen centered free radicals on human low density lipoprotein. Biochem J 1989; 262: 707-712.

8 Wolff S, Garner A, Dean R. Free radicals, lipids and protein breakdown. Trends Biochem Sci 1986; 11: 27-31.

9 McKelvey TG, Hollwarth ME, Granger DN, Engerson TD, Landler U, Jones HP. Mechanisms of conversion of xanthine dehydrogenase to xanthine oxidase in ischemic rat liver and kidney. Am J Physiol 1988; 254: G753-G760.

10 Berman RS, Martin W. Arterial endothelial barrier dysfunction: actions of the homocysteine and hypoxanthinexanthine oxidase free radical generating system. $\mathrm{Br} J$ Pharmacol 1993; 108: 920-926.

11 Butler R, Morris A, Belch J, Hill A, Struthers A. Allopurinol normalizes endothelial dysfunction in type 2 diabetics with mild hypertension. Hypertension 2000; 35: 746-751.

12 Farquharson C, Butler R, Hill A, Belch J, Struthers A. Allopurinol improves endothelial dysfunction in chronic heart failure. Circulation 2002; 106: 221-226.

13 Magalang J, Dmochowski J, Veeramachaneni S, et al. Prediction of the apnea-hypopnea index from overnight pulse oximetry. Chest 2003; 124: 1694-1701.

14 Yagi K. Assay for serum lipid peroxide level and its clinical significance. In: Yaki K, ed. Lipid Peroxides in Biology and Medicine. New York, Academic Press, 1982; pp. 223-242.

15 Shahar E, Whitney CW, Redline S, et al. Sleep-disordered breathing and cardiovascular disease cross-sectional results of the Sleep Heart Health Study. Am J Respir Crit Care Med 2001; 163: 19-25.

16 Saito T, Yoshikawa T, Sakamoto $\mathrm{Y}$, Tanaka $\mathrm{K}$, Inoue $\mathrm{T}$, Ogawa R. Sleep apnea in patients with acute myocardial infarction. Am J Respir Crit Care Med 1991; 19: 938-941.

17 Celermajer DS, Sorensen KE, Gouch VM, et al. Non invasive detection of endothelial dysfunction in children and in adults at risk of atherosclerosis. Lancet 1992; 340: 1111-1115.

18 Carlson JT, Rangemark C, Hedner JA. Attenuated endothelium-dependent vascular relaxation in patients with sleep apnea. J Hypertens 1996; 14: 577-584.

19 Nieto F, Herrington D, Redline S, Benjamin E, Robbins J. Sleep apnea and markers of vascular endothelial function in a large community sample of older adults. Am J Respir Crit Care Med 2004; 169: 354-360.

20 Ip M, Tse H, Lam B, Tsang K, Lam W. Endothelial function in obstructive sleep apnea and response to treatment. Am J Respir Crit Care Med 2004; 169: 348-353.

21 Zweier J, Broderick R, Kuppusamy P, ThompsonGorman S, Lutty G. Determination of the mechanism of free radical generation in human aortic endothelial cells exposed to anoxia and re-oxygenation. J Biol Chem 1994; 269: 24156-24162.

22 Cardillo C, Kilcoyne CM, Cannon RO 3rd, Quyyumi AA, Panza JA. Xanthine oxidase inhibition with oxypurinol improves endothelial vasodilator function in hypercholesterolemic but not in hypertensive patients. Hypertension 1997; 30: 57-63.

23 Ohara Y, Peterson TE, Harrison DG. Hypercholesterolemia increases endothelial superoxide anion production. J Clin Invest 1993; 91: 2546-2551.

24 Steinberg HO, Chaker H, Leaming R, Johnson A, Brechtel G, Baron AD. Obesity/insulin resistance is associated with endothelial dysfunction. Implications for the syndrome of insulin resistance. J Clin Invest 1996; 97: 2601-2610.

25 Arcaro G, Zamboni M, Rossi L, et al. Body fat distribution predicts the degree of endothelial dysfunction in uncomplicated obesity. Int J Obes Relat Metab Disord 1999; 23: 936-942.

26 Perticone F, Ceravolo R, Candigliota M, et al. Obesity and body fat distribution induce endothelial dysfunction by oxidative stress: protective effect of vitamin C. Diabetes 2001; 50: 159-165.

27 de Jongh RT, Serne EH, IJzerman RG, de Vries G, Stehouwer CD. Impaired microvascular function in obesity: implications for obesity-associated microangiopathy, hypertension, and insulin resistance. Circulation 2004; 109: 2529-2535.

28 Nakazono K, Watanabe N, Matsuno K, Sasaki J, Sato T, Inoue M. Does superoxide underlie the pathogenesis of hypertension? Proc Natl Acad Sci USA 1991; 88: 10045-10048.

29 Schulz R, Schmidt D, Blum A, et al. Decreased plasma levels of nitric oxide derivatives in obstructive sleep apnea: response to CPAP therapy. Thorax 2000; 55: 1046-1051.

30 Ip M, Lam B, Chan $L$, et al. Circulating nitric oxide is suppressed in obstructive sleep apnea and is reversed by nasal continuous positive airway pressure. Am J Respir Crit Care Med 2000; 162: 2166-2171.

31 Barcelo A, Miralles C, Barbe F, Vila M, Pons S, Agusti A. Abnormal lipid peroxidation in patients with sleep apnea. Eur Respir J 2000; 16: 644-647.

32 Farquharson C, Struthers A. Spironolactone increases NO bioactivity, improves endothelial vasodilator dysfunction, and suppresses vascular AI/AII conversion in patients with CHF. Circulation 2000; 101: 594-597.

33 Egashira K, Hirooka Y, Kai H, et al. Reduction in serum cholesterol with pravastatin improves endotheliumdependent coronary vasomotion in patients with hypercholesteremia. Circulation 1994; 89: 2519-2524.

34 Hijmering M, Stroes E, Pasterkamp G, Sierevogel M, Banga J, Rabelink T. Variability of flow mediated dilation: consequences for clinical application. Atherosclerosis 2001; 157: 369-373.

35 Nielsen F, Mikkelsen B, Nielsen J, Andersen $\mathrm{H}$, Grandjean P. Plasma malondialdehyde as biomarker for oxidative stress: reference interval and effects of life-style factors. Clin Chem 1997; 43: 1209-1214.

36 Guthikonda S, Sinkey C, Barenz T, Haynes W. Xanthine oxidase inhibition reverses endothelial dysfunction in heavy smokers. Circulation 2003; 107: 416-421. 